En 2005, le comité de pilotage de l'Année mondiale de la physique en France avait mandaté l'Espace des Sciences de Rennes ${ }^{(1)}$ et I'Université de Rennes 1 pour réaliser une " valise énergie » susceptible d'éclairer les élèves des collèges sur ce qu'est l'énergie sous toutes ses formes et ce qu'elle sera à I'avenir.

La conception de cette valise a nécessité beaucoup d'efforts ; nous avons en effet choisi de nous appuyer sur une approche essentiellement expérimentale même si, dans certains cas, l'appui sur des vidéos nous a semblé judicieux.

Une variante de cette valise énergie a été présentée lors d'une semaine scientifique itinérante, dans huit grandes villes de Madagascar.

\section{Les valises de Madagascar}

Daniel Bideau (daniel.bideau@univ-rennes1.fr)

Institut de Physique de Rennes, Bâtiment 11A, Université de Rennes 1, 263 av. du Général Leclerc, 35042 Rennes Cedex

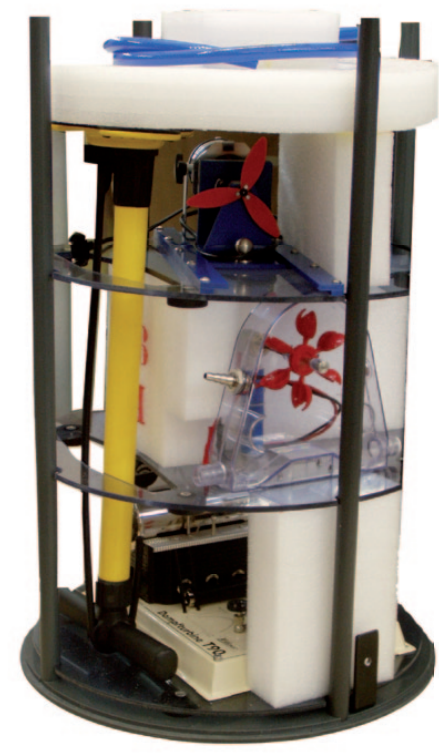

La valise :

un outil pour familiariser les élèves avec l'énergie

Le but de cette réalisation était :

- d'initier les élèves à l'énergie via l'observation et l'expérimentation ;

- de leur faire comprendre, à travers des manipulations simples, les notions fondamentales liées à l'énergie (définition, formes d'énergie et transformations, énergies fossiles et renouvelables, travail, chaleur, changement d'état...). Une voiture à ressorts permet d'illustrer le stockage de l'énergie et l'énergie potentielle ;

- de les sensibiliser aux problèmes énergétiques actuels engendrés par une consommation excessive des ressources fossiles, et à leur impact sur l'environnement ;

- de les responsabiliser dans leur comportement quotidien afin de les conduire à limiter tout gaspillage ;

- de leur présenter un aperçu des recherches menées actuellement pour préserver notre environnement et développer l'utilisation des énergies renouvelables à moyen terme.

Les expériences utilisent souvent des objets de la vie courante, voire des jouets : une simple lampe électrique à action manuelle permet de mettre en évidence la transformation d'énergie musculaire en énergie mécanique, puis en énergie électrique et enfin en lumière. Les encadrés illustrent deux " expériences ». L'encadré 1 montre l'intérêt de l'isolation thermique des maisons. L'encadré 2 présente le " clou " de ces dispositifs : une machine à vapeur, qui est en fait un jouet pédagogique acheté en Allemagne.

L'ensemble de ces petites expériences (fig. 1A) est contenu dans deux coffrets cylindriques d'environ un mètre de haut et $50 \mathrm{~cm}$ de diamètre, ayant la forme d'une pile électrique (fig. 1B). Les différentes expériences sont rangées dans ces cylindres, de façon à en optimiser l'occupation.
1. La « valise énergie ». A : Le contenu d’un des deux coffrets. En bas : la machine à vapeur ; au milieu : une " éolienne ", qui peut être entraînée par la pompe à vélo jaune ; en haut : un ventilateur, qui est actionné à partir de l'énergie électrique d'une cellule photovoltaïque. B : Aspect extérieur d'un coffret. 
Cette réalisation a reçu le Prix Diderot 2007 de l'initiative culturelle, à l'occasion du $25^{\mathrm{e}}$ congrès de l'Amcsti (Association des Musées et Centres pour le développement de la Culture Scientifique, Technique et Industrielle).

Depuis 2005, elle a été montrée dans différentes manifestations scientifiques, et elle circule dans les écoles, en particulier sous l'égide de l'Espace des Sciences de Rennes ou de sa "succursale " Espace des Sciences du Pays de Morlaix. Elle est finalement bien adaptée au public CM1-CM2 et collège, classes où l'énergie fait partie du programme scolaire. Elle convient également bien à des animations scientifiques, comme nous le verrons ci-dessous. Il est possible de l'acheter, sous une forme améliorée depuis (elle est en particulier plus facile à transporter) et à un prix raisonnable, auprès de l'Espace des Sciences de Rennes. (www.espace-sciences.org/archives/valise-energie)

\section{Une semaine scientifique à Madagascar}

En automne 2006, lors d'un séjour aux États-Unis, j'ai été contacté par une ONG qui a son siège près de Rennes, l'association «Défi » (www.ongdefi.org). Elle se donne pour but la mise en place d'un programme de «Renforcement des Sciences et Techniques dans l'Enseignement Primaire » (RSTEP), destiné à améliorer le contenu et la qualité des enseignements reçus par les enfants des pays du Sud, en particulier Madagascar et Haïti. Ses membres, bénévoles ou salariés, sont des enseignants ou des animateurs. La spécificité de ce programme est d'apporter, aux instituteurs et autres enseignants, des formations qui renforcent leurs connaissances en sciences et techniques, matières souvent négligées (surtout l'aspect expérimental) dans leurs cursus de formation et qui sont néanmoins très importantes pour faire entrer un pays pauvre dans le monde moderne. "La main à la pâte " a été et reste une source d'inspiration pour Défi. Depuis l'an 2000, environ 11000 enseignants et formateurs d'enseignants ont été formés : 2 millions d'enfants bénéficiaires. $\mathrm{La}$ valise énergie, dans la mesure où elle repose essentiellement sur une approche expérimentale, les a intéressés.

L'idée qui m'était soumise était de participer à une action de type "Culture scientifique ». Il s'agissait de mettre en place une semaine scientifique itinérante, dans huit grandes villes de Madagascar (une semaine par ville), l'animation étant assurée par trois associations (deux associations malgaches et Défi). Je devais, pour ma part, participer à la première de ces semaines en animant à Tuléar, au sud de Madagascar, un atelier (fig. 2) autour de la valise énergie, mon voyage et mon séjour étant financés par Défi. Au programme : informatique, sciences de la vie et de la Terre, physique, chimie et environnement. Au-delà de ma tâche d'animation, mon rôle était de former à la valise énergie un animateur de Défi qui devait ensuite, durant les sept semaines suivantes, présenter cette valise.

Cette animation itinérante a connu un grand succès : la ville de Mahajanga (ouest de Madagascar) a accueilli 3100 visiteurs en 5 jours. Les échanges avec les jeunes Malgaches ont été intéressants. Le fait de pouvoir eux-mêmes faire les expériences était nouveau et captivant pour ces jeunes scolaires, qui restaient longuement sur les stands. Signalons néanmoins quelques épisodes inattendus.

Une des "manips " de la valise consiste à comparer les temps de chauffage et la température limite de deux petites maisons équipées de "radiateurs " identiques, dont l'une est isolée et l'autre pas (encadré 1). Problème : ce dispositif est très pédagogique en Bretagne, mais pas à Madagascar où une climatisation est plus utile qu'un chauffage. Nous avons été amenés à modifier cette manip dans une valise spéciale "Afrique ", en plaçant les sources de chauffage à l'extérieur (le soleil). Autre problème : nos animations étaient prévues pour des écoliers. La presse locale en a fait état. Je fus surpris de recevoir à plusieurs reprises la visite d'étudiants et même d'enseignants de l'université locale, qui venaient me demander si j'étais capable de leur faire parvenir des livres de physique, voire du matériel d'expérimentation ${ }^{(2)}$.

Cette expérience a été enrichissante. Avec la SFP (Sylvie Magnier), nous avons essayé de la prolonger dans d'autres pays africains, en intervenant plus directement dans les écoles. La main d'œuvre existe (mes copains retraités ou émérites). Le matériel est prêt, financé par le ministère de l'Éducation. Malheureusement, le soutien attendu du ministère des Affaires étrangères, qui devait financer les déplacements et séjours, n’a pas suivi...

Mais Défi, qui vient de fêter ses 20 ans, continue ses activités, en particulier à Haïti et au Bénin. -

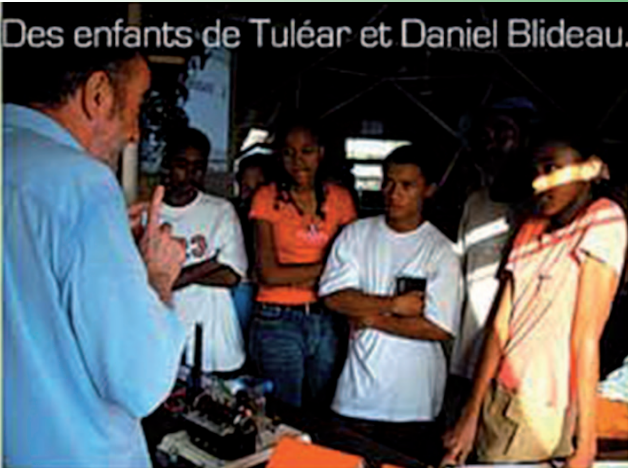

2. « Atelier énergie » à Tuléar

\section{Isolation thermique} des habitations

Le dispositif présenté dans la figure ci-dessous a pour objet d'illustrer la nécessité $d^{\prime}$ isoler thermiquement les habitations.

Ces deux « maisons » sont de même forme et taille. L'une est isolée (par du bois), l'autre non. Le toit est mobile pour permettre de voir l'intérieur (rien de magique). Une lampe installée au centre de chacune d'entre elles constitue le chauffage. L'augmentation de la température et sa valeur finale sont visibles de l'extérieur.

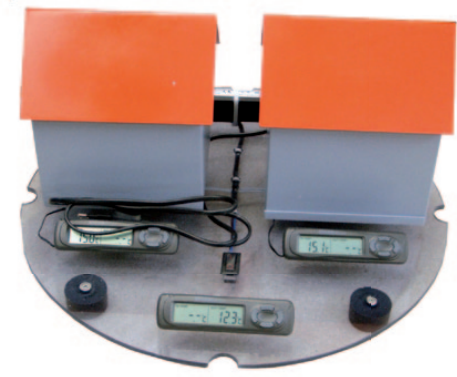

\section{La machine à vapeur}

Encadré 2

La source primaire est un « allume barbecue ». Celui-ci fournit la chaleur à la machine à vapeur (cuve) qui fait tourner une turbine. Une petite dynamo, actionnée par la turbine permet d'alimenter une petite lampe : fiat lux !

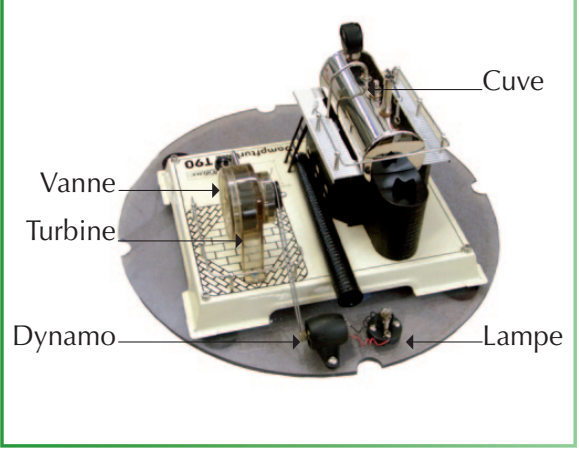

